\title{
Highly-accurate gesture recognition based on ResNet with low-budget data gloves
}

\author{
Hongrui Min \\ hr.min@siat.ac.cn \\ University of Chinese Academy of \\ Sciences \\ China \\ Shenzhen Institute of Advanced \\ Technology, CAS \\ Shenzhen, China \\ Xiaorui Tian \\ tfjstxr@gmail.com \\ Shenzhen University \\ Shenzhen, China
}

\author{
Chao Chen \\ chao.chen@siat.ac.cn \\ Shenzhen Institutes of Advanced \\ Technology, CAS \\ Shenzhen, China \\ Yongkui Yang \\ yk.yang@siat.ac.cn \\ Shenzhen Institute of Advanced \\ Technology, CAS \\ Shenzhen, China
}

\author{
Shixin Huang \\ huangsx@siat.ac.cn \\ Shenzhen Institute of Advanced \\ Technology, CAS \\ Shenzhen, China
}

\author{
Zheng Wang* \\ zheng.wang@siat.ac.cn \\ Shenzhen Institute of Advanced \\ Technology, CAS \\ Shenzhen, Guangdong, China
}

\begin{abstract}
Gesture recognition has been widely used in various fields such as virtual reality (VR), human-computer interaction, and clinical medicine. Traditionally, gesture recognition requires expensive sensors to obtain high-precision data, but some applications-such as VR games-do not require high accuracy and users prefer a lower cost instead. Thus some researchers began to use Convolutional Neural Networks (CNN) to recognize gestures directly without other calibration equipment. However, using $\mathrm{CNN}$ encounters the degradation phenomenon of the neural network. In this paper, we propose a highly-accurate gesture recognition method for lowprecision glove data based on Residual neural Networks (ResNet). Our method combines 1D convolution with 2D convolution and adopts a sliding window for sample size constraint to achieve data enhancement. Evaluation results indicate that compared to the $\mathrm{CNN}$ method, our method improves the classification accuracy rate from $94.4 \%$ to $99.2 \%$.
\end{abstract}

\section{CCS CONCEPTS}

- Computing methodologies $\rightarrow$ Model development and analysis; Modeling methodologies.

\section{KEYWORDS}

gesture recognition; low-budget data glove; VR games; ResNet

ACM Reference Format:

Hongrui Min, Chao Chen, Shixin Huang, Xiaorui Tian, Yongkui Yang, and Zheng Wang. 2021. Highly-accurate gesture recognition based on

"Zheng Wang is the corresponding author (zheng.wang@siat.ac.cn).

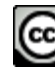

This work is licensed under a Creative Commons Attribution International 4.0 License.

AISS 2021, Nov. 26-28, 2021, Sanya, China

(C) 2021 Association for Computing Machinery.

ACM ISBN 978-1-4503-8586-2/21/11.

https://doi.org/10.1145/3503047.3503153
ResNet with low-budget data gloves. In 20213 rd International Conference on Advanced Information Science and System(AISS 2021), Nov. 26-28, 2021, Sanya, China. ACM, New York, NY, USA, 6 pages. https://doi.org/10.1145/ 3503047.3503153

\section{INTRODUCTION}

Nowadays, gesture recognition has a pivotal role in various fields such as human-computer interaction [20], robotics [19], self-driving [13],smart wearable device [25] and virtual reality [24]. Seokwon et al. [15] describe the application of data gloves in VR games. In VR games, many things that are difficult to accomplish in real life can be easily achieved. You can play the piano, ski, and even save the world like the protagonist of a Marvel movie. All of this just requires one compact VR equipment. However, the cost is a classic problem in the application of VR equipment. Good-quality and inexpensive equipment is one of the necessary conditions for the promotion of VR games. VR games are mainly composed of interactive and action simulation which need a lot of motion recognition. It has been observed that compared with wireless controllers, vision-based gesture recognition lacks the sense of vibration feedback. Also, it is impossible to achieve satisfactory sensitivity and accuracy by relying on computer vision alone. Fortunately, low-budget data gloves can overcome these shortcomings. At present, gesture recognition with data gloves can be used for some common operations, such as aiming, selecting, moving, rotating, zooming, etc. Virtual objects can be directly touched with users' hands, and data gloves can provide users with tactile feedback.

In addition, gesture recognition with data gloves does not require placing hands in a specific position to track and identify. In many complex game scenarios, we also need to do different actions in different places. Data gloves are not restricted by light, camera angle of view, distance, and other factors. Compared with other devices, VR devices with data gloves can provide a better immersive experience. Data gloves can expand the application scenarios of VR, especially those that require finger details, such as teaching experiments. Ognjan et al. [18] have also researched low-budget data gloves, but in his work, the type of gestures is very limited. 
Quam et al. [22] have researched that gesture recognition can be accomplished by the data gloves with flex sensors. Then some researchers use neural networks [27] to study gesture recognition. In terms of classification, neural networks are being increasingly adopted by researchers. In theory, neural networks can fit any continuous function and have powerful feature extraction capabilities. Gesture recognition with data gloves has been widely researched via a neural network like Convolutional Neural Network (CNN) [17] and Recurrent neural network(RNN) [10]. Weissmann et al. [29] propose a gesture recognition method based on neural networks used in VR applications. Emmanuel et al. [3] use the CNN classification with a weft knit data glove on grasp recognition and achieve the mean accuracy of up to $86.27 \%$. Sai et al. [11] propose a Fully-Connected Neural Network (FCNN) classifier which could learn hand gestures from data with $98 \%$ accuracy but only a few grasp movements are evaluated. The more complex the neural network is, the more parameters there are. For example, GPT-3 [4] has hundreds of billions of parameters. It is infeasible to deploy it on a portable device like a glove. Convolutional neural networks are more suitable for data gloves than other neural networks because of shared weights. In this work, we refer to ResNet [9] and link the $\mathrm{CNN}$ across layers to recognize gestures and make the comparison with machine learning algorithms.

On the one hand, this paper reveals that it is possible to recognize different common gestures accurately from inertial sensor data with a pair of low-budget data gloves. Using a residual neural network instead of $\mathrm{CNN}$ to classify gestures generates fresh insight that inexpensive data gloves can help us experience VR. On the other hand, we evaluate the performance of the classification method based on ResNet in the relatively small data set, which proves that our method generates good results.

In summary, the following contributions are made in this paper:

- Motivated by ResNet, we design a new deep learning model which combines $1 \mathrm{D}$ convolution with $2 \mathrm{D}$ convolution.

- Due to the limitation of sample size, we adopt a sliding window method to achieve data enhancement.

- We demonstrate that our approach significantly improves the maximum accuracy of common gesture recognition up to $99.2 \%$, while the accuracy of $\mathrm{CNN}$ is only $94.4 \%$ under the same conditions.

\section{METHOD}

\subsection{Dataset}

In this paper, we designed a pair of data gloves to collect the corresponding data from subjects. The gestures are given by the Ninapro [2] which is famous for EMG databases. The data gloves we designed consist of 6 inertial sensors and each sensor contains an MPU-6050 gyroscope. The sensor can record the joint angle information of the fingers. It is driven by an ARM architecture Micro Controller Unit (MCU). The data glove is pretty cheap and convenient.

Grasping movement is as common as sign languages in our daily lives. Thus we selected 13 kinds of common gestures including sign languages and grasping movements. When collecting data, we always keep the palm in the vertical direction. Then we use a data glove to directly record relevant joint signals at a frequency of 30 hertz by the 6 sensors. For each gesture, we will record 500 values per sensor. The raw hand gesture data will be used as the input data of the neural network after the sliding window. Figure 2 shows all the common gestures in this experiment. It's important to note that our method does not need to map from sensor values to actual joint angles. It means that a complex calibration is not necessary. So our method can quickly recognize common human hand gestures. The appearance of the data glove is shown in Figure 1.

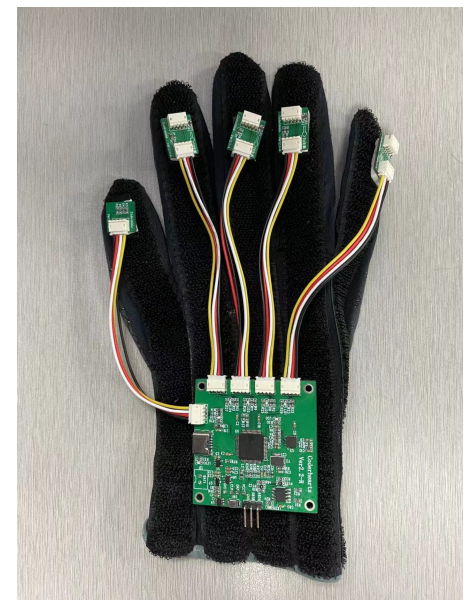

Figure 1: Low-budget data glove used in the experiments.

\subsection{Data preprocessing}

Most research uses the original data as input directly without considering data preprocessing. If the collected raw data is directly input into the neural network, the final result will likely be affected by factors such as noise. We must preprocess the data. Inspired by previous bio-signal processing methods[7] [5], we adopt a sliding window method to process the raw gesture data. The joint angle data of each gesture we collected is a series of continuous signals. The collected signal can be represented as following:

$$
A=\left\{a_{1}, a_{2}, \ldots, a_{n}\right\} .
$$

Traditional approach is to combine a single vector $a_{k}$, we adopt a sliding window to intercept the collected data, the result is represented as:

$$
B_{1}=\left\{a_{1}, a_{2}, \ldots a_{t}\right\}, t<n .
$$

Like the convolution kernel in the convolution neural network, the data is intercepted by moving the window according to the given step size $s$, the next result can be presented as:

$$
B_{2}=\left\{a_{1+s}, a_{2+s}, \ldots a_{t+s}\right\}, t<n .
$$

The data processed by the sliding window will be as input to the neural network. It not only enhances the original data but also the generalization performance of the network. The principle of the sliding window is shown in Figure 3.

\subsection{Traditional algorithms}

Traditional methods on gesture recognition such as decision trees [6], random forests, and linear regression have made significant 

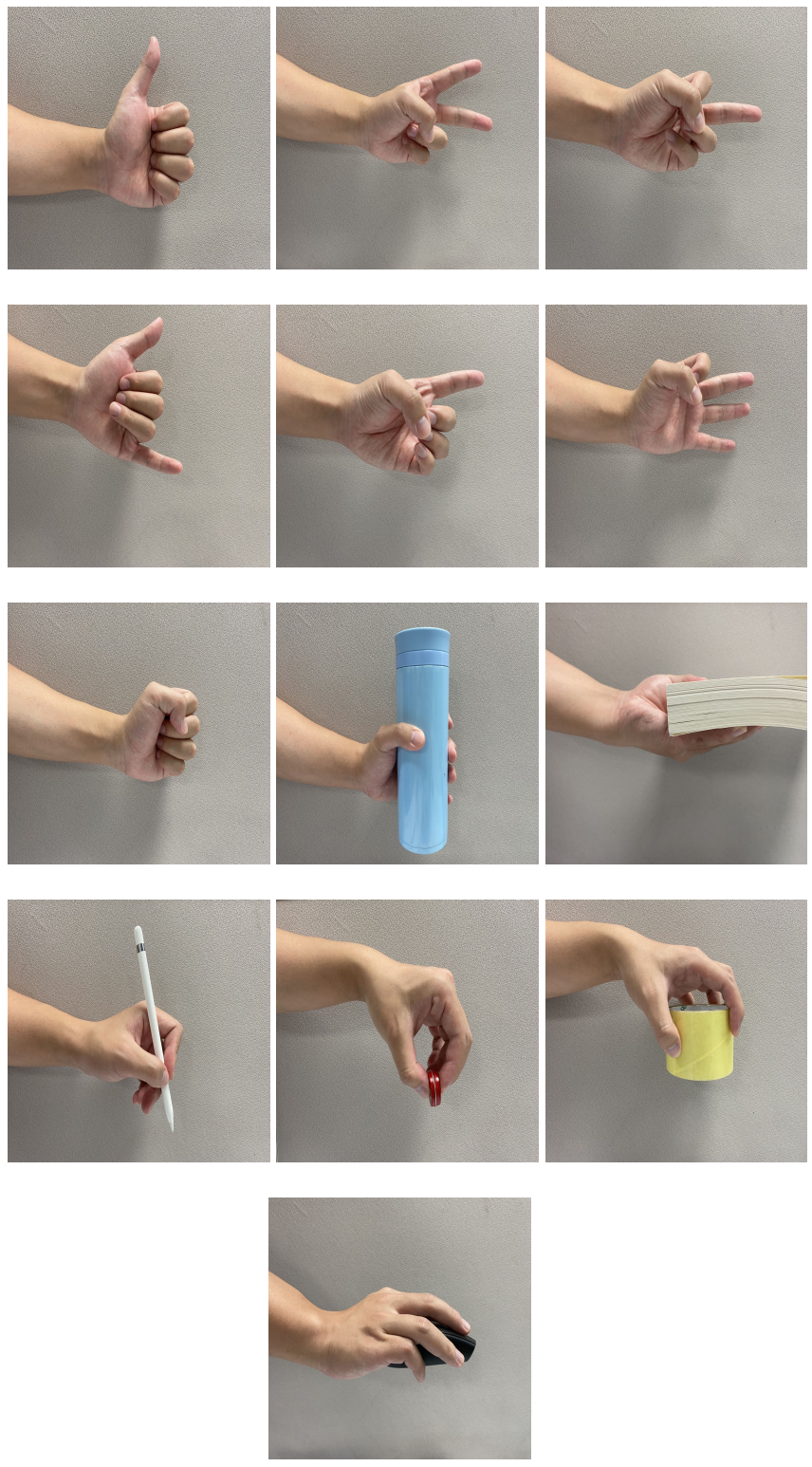

Figure 2: Different gestures used in the experiments.

contributions to gesture recognition. Each of them has its advantages and disadvantages. We will introduce these common gesture recognition algorithms as follows:

- Linear regression (LR) is simple and easy to understand. It can process numerical data and predict that the target data is continuous. Gesture data is just a kind of continuous data. So lots of researchers have been studied gesture recognition with linear regression. LR can be calculated as follows:

$$
f(x)=w^{T} x+b .
$$

We can directly see the degree of influence of each feature on the result from the weight $w$. However, there are still

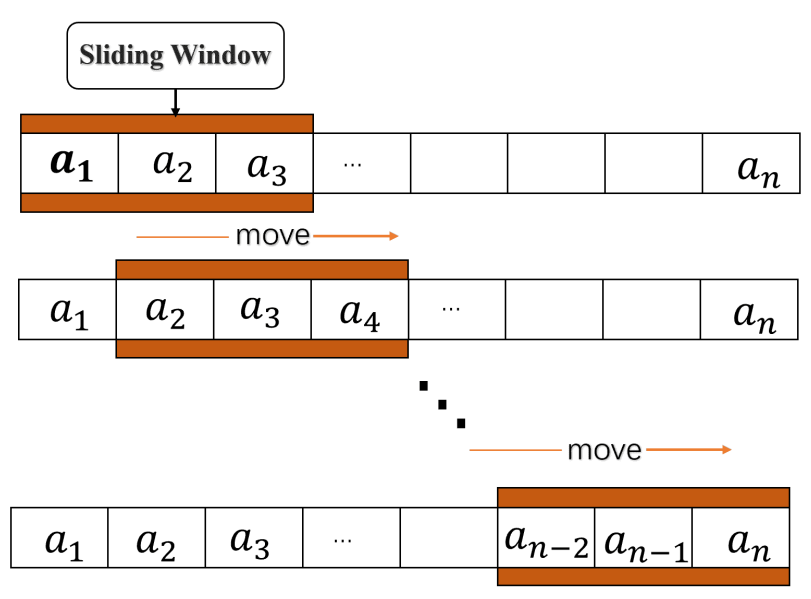

Figure 3: Schematic diagram of the sliding window.

some limits. For example, not all models are linear and LR cannot handle non-linear models effectively. Unfortunately, this is one of the inevitable situations in the research.

- Decision tree (TR) is a very classic machine learning method. In many classification problems, especially when the amount of data is relatively small, decision trees usually perform very well than any other machine learning method. Decision tree training requires fewer data and is highly interpretable and that is why we chose it as the method of comparison. But TR is prone to produce an overly complex model, resulting in poor generalization performance. At the same time, learning an optimal decision tree is usually an NP-hard problem.

- Random forest (RF) is a classifier built haphazardly and contains multiple decision trees. It can handle very highdimensional data without feature selection. In the training process, the mutual influence between features can be detected and after training, it can give out which features are more important. When RF solves the regression problem, it does not perform as well as it does in classification. This is because it does not give a continuous output. These machine learning methods have limitations in the field of gesture recognition.

- Convolutional neural networks are widely used in the field of deep learning, and many classification problems can be easily solved through it. Compared with other neural networks, $\mathrm{CNN}$ can automatically perform feature extraction, and sharing weights. It means that the number of parameters will be relatively small, which helps us deploy neural networks on devices with low computing power such as gloves. However, if the network level is too deep, the gradient disappears or explodes. Therefore, there is still room for improvement in many related studies.

\subsection{The framework of the proposed gesture recognition}

After the neural network was proposed, many scholars have been trying to deepen the number of layers of the network. From the 
initial three-layer fully connected neural network to AlexNet [14], VGG16 [26], etc., the number of neural networks is increasing. But the researchers soon discovered that when the number of layers was increased to a certain number of layers, the accuracy began to drop. The emergence of a Residual neural network (ResNet) solves this problem. ResNet has been widely used in deep learning algorithms and make a breakthrough. If we only use CNN to classify gestures, we usually need a multi-layer neural network. However, the experimental results will not increase with the depth of the neural network, and the accuracy rate will start to decline after the neural network exceeds a certain depth. Compared with previous deep learning algorithms, ResNet can effectively avoid the gradient disappearance or gradient explosion, which is especially important when the neural network is deep. We decide to change the original convolutional neural network structure and added a residual block. Figure 4 shows our design of the residual block.

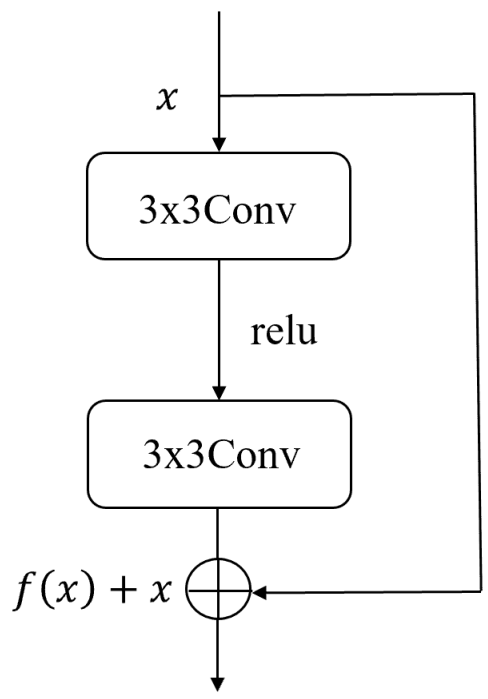

Figure 4: Residual block.

We conduct the experiments on the PyTorch [12] platform and scikit-learn package [21]. Our algorithm flow chart is shown in Figure 5. After we collected data through the data glove, then we process the gesture data by sliding window. Next, the data will be sent to the neural network for classification. Our network has a total of 14 layers, including 4 residual modules. We choose the cross-entropy as a loss function and use the Adam optimizer. For multi-class problems, the softmax function is one of the best choices. It can be calculated as follow:

$$
\text { Soft max }\left(x_{i}\right)=\frac{\exp \left(x_{i}\right)}{\sum_{j} \exp \left(x_{j}\right)} .
$$

\section{RESULT}

The results of different classification methods are illustrated in Figure 6. It can be seen that on average, gesture recognition method based on ResNet has higher accuracy than any other method. From

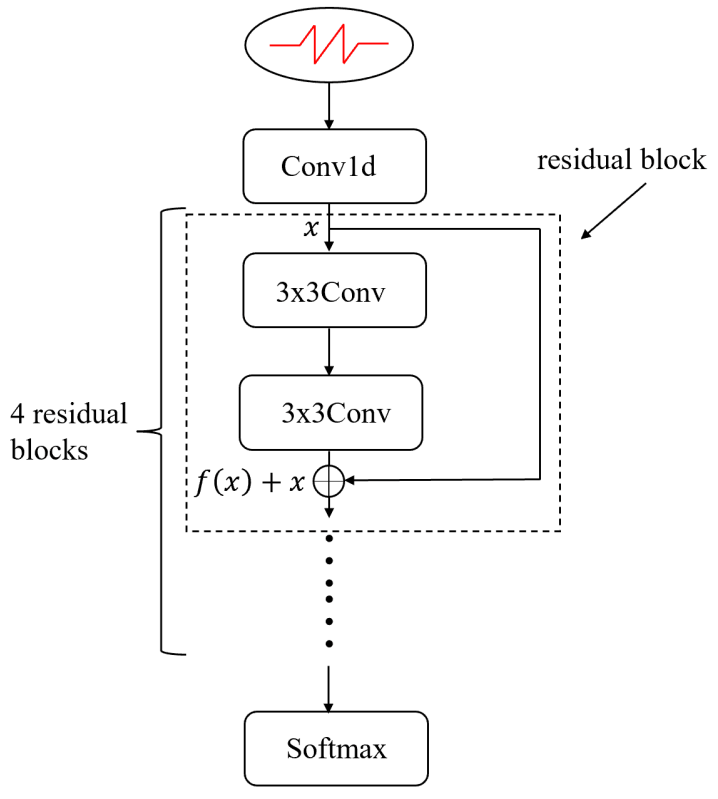

Figure 5: The architecture of the proposed model.

Figure 6, we can easily observe that the results based on ResNet and random forest (RF) [1] both achieve promising results in terms of precision. Decision tree (TR) performs worst in general because decision tree has a high risk of over-fitting. We also note that the CNN model shows worse performance than the based on the ResNet model as well. We argue this may be caused by the fact that the input data size is relatively small. Compared with random forest (RF), linear logistic regression (LR) [8], and other machine learning methods, the CNN model is not adequate. In summary, we have proved that gesture recognition based on ResNet could recognize different common gestures accurately from initial inertial sensor input with a pair of low-budget data gloves. Further classification results could be observed through the confusion matrix.

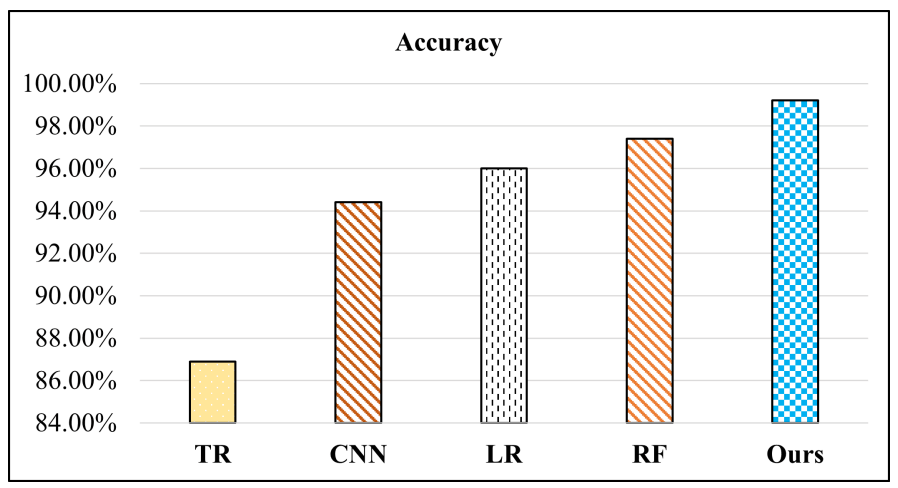

Figure 6: The results of different classification methods, where the $x$-axis represents the method and the $y$-axis denotes the accuracy. 
The confusion matrix [28] which stands for estimation accuracy with each gesture is shown in Figure 7. From the confusion matrix, we find that sign languages are easier to identify than grasp movement. Due to the relatively large amount of data, it is not intuitive to draw the confusion matrix directly. Therefore, after normalizing the data, we keep two decimal places and use the sklearn package to draw the confusion matrix. From Figure 7, we can observe the classification results. Because we always wear the data glove and keep the palm in the vertical direction to acquire data, so the gestures of label 1 and label 12 are very similar. The same is true for label 5, label 9, and label 10 gestures. A very small number of data with labels 0 and label 6 is not classified by neural networks. For these similar gestures, our model based on the ResNet is still able to reach at least $96 \%$ accuracy. This proved that ResNet has high estimation accuracy in gesture recognition even if there are extremely similar gestures. For those gestures with big differences, the model based on the ResNet makes few mistakes.

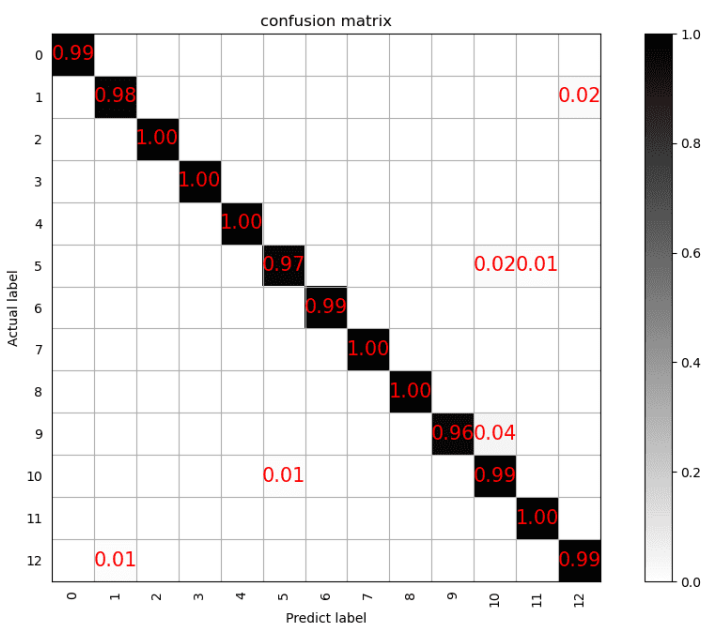

Figure 7: The confusion matrix

\section{DISCUSSION}

Gesture recognition has been widely used in daily lives. Using a pair of low-budget data gloves could easily realize gesture recognition. Data gloves can help us complete many tasks in human-computer interaction scenarios. Correctly and quickly recognizing the users' gestures can help us judge theirs' motivation which plays an important role in VR games [23] and other scenarios. In this study, we researched hand gesture recognition based on ResNet and compare it with traditional machine learning methods.

Generally speaking, the volume of data collected by data glove is not large. The most exciting finding was that $\mathrm{CNN}$ sometimes is not effective compared with random forest, linear logistic regression, and other machine learning methods. Machine learning algorithms also have a good performance when the data set is relatively small and is superior to lots of neural network classification algorithms. Thus, in this research, we choose to improve the original CNN model and combine the residual block and the result turns out that the improved model is more suitable than CNN. For similar gestures,
$\mathrm{CNN}$ needs to deepen the number of layers to distinguish due to small data, but worsening the number of network layers will cause the gradient to disappear or the gradient to explode. Combined with ResNet can overcome these shortcomings so our deep learning model combined 1D convolution with 2D convolution not only can the training time of the multilayer neural network be reduced, but also a very high accuracy rate can be achieved.

However, there are still some shortcomings in the work we have done. Firstly, we do not acquire continuous motion gesture data and this is will be one of our works. The gesture data is basic functionality without complex gesture data. And continuous motion gesture is necessary for gesture recognition. Only the continuous motion gesture can be accurately estimated, low-budget data gloves can play an irreplaceable role in VR games, human-computer interaction, and other scenarios. In terms of gesture recognition with data gloves, continuous motion gesture estimation is a trend [16]. Secondly, the amount of gesture data acquired in our work is not big enough. We should consider different subjects in terms of gender, age and health.

To some extent, gesture data is a type of EMG. This means it is also a type of time series data. For this kind of data, we can also use recurrent neural networks (RNN) to process. In this field, few people currently use RNN alone to recognize continuous motion gesture data with a pair of data gloves. For continuous motion gesture data, long short-term memory (LSTM) neural network may be a solution [30].

As discussed above, our future work will focus on continuous motion gestures on various subjects. We will try to do continuous motion gesture recognition based on LSTM.

\section{CONCLUSION}

The purpose of this paper is to determine whether ResNet could classify gesture data in the case that the amount of data is relatively small. The result is in line with our expectations. This is of great significance to the promotion of VR. Deploying a neural network with a relatively small amount of parameters on the chip can quickly and accurately identify various gestures. At the same time, low-cost gloves can be widely used in many human-computer interaction scenarios as wearable devices.

The paper has argued that low-budget data gloves could recognize gestures easily and effectively. In terms of some applications, especially in VR games, the lower cost is vital. In this paper, we propose a method based on ResNet via a sliding window to recognize the gesture. The model architecture is not extremely complex but the result is very promising. Simple neural networks can be deployed on low-end chips and this is meaningful. We experimented with CNN, RF, LR, and TR classifiers to identify which one is the most suitable for gesture recognition. Our method achieves the best prediction gesture result with $99.2 \%$ accuracy on the test data. In the future, We plan to increase the number of gestures including gestures to cover the general gestures in VR games based on our low-budget data-glove. Although the classification accuracy of our algorithm has achieved $99.2 \%$, it is still limited in the object seen scenarios and there is no continuous dynamic gesture. Future work will involve continuous dynamic gesture and gesture recognition in unseen scenarios. 


\section{ACKNOWLEDGMENTS}

This research was funded by the National Natural Science and Foundation of China (NSFC 61902355), the Key-Area Research and Development Program of Guangdong Province (grant number 2019B010155003), and the Guangdong Basic and Applied Basic Research Foundation (grant number 2020B1515120044).

\section{REFERENCES}

[1] Jehad Ali, Rehanullah Khan, Nasir Ahmad, and Imran Maqsood. 2012. Random Forests and Decision Trees. www.IJCSI.org

[2] Manfredo Atzori, Arjan Gijsberts, Claudio Castellini, Barbara Caputo, Anne Gabrielle Mittaz Hager, Simone Elsig, Giorgio Giatsidis, Franco Bassetto, and Henning Müller. 2014. Electromyography data for non-invasive naturally-controlled robotic hand prostheses. Scientific Data 1 (12 2014). https://doi.org/10.1038/ sdata.2014.53

[3] Emmanuel Ayodele, Tianzhe Bao, Syed Ali Raza Zaidi, Ali M. A. Hayajneh, Jane Scott, Zhi-Qiang Zhang, and Des McLernon. 2021. Grasp Classification With Weft Knit Data Glove Using a Convolutional Neural Network. IEEE Sensors fournal 21, 9 (2021), 10824-10833. https://doi.org/10.1109/JSEN.2021.3059028

[4] Tom B. Brown, Benjamin Mann, Nick Ryder, Melanie Subbiah, Jared Kaplan, Prafulla Dhariwal, Arvind Neelakantan, Pranav Shyam, Girish Sastry, Amanda Askell, Sandhini Agarwal, Ariel Herbert-Voss, Gretchen Krueger, Tom Henighan, Rewon Child, Aditya Ramesh, Daniel M. Ziegler, Jeffrey Wu, Clemens Winter, Christopher Hesse, Mark Chen, Eric Sigler, Mateusz Litwin, Scott Gray, Benjamin Chess, Jack Clark, Christopher Berner, Sam McCandlish, Alec Radford, Ilya Sutskever, and Dario Amodei. 2020. Language Models are Few-Shot Learners. (5 2020). http://arxiv.org/abs/2005.14165

[5] Chao Chen, Weiyu Guo, Chenfei Ma, Yongkui Yang, Zheng Wang, and Chuang Lin. 2021. sEMG-based Continuous Estimation of Finger Kinematics by Large Scale Temporal Convolutional Network. (2021), 1-13.

[6] Gaolin Fang, Wen Gao, and Debin Zhao. 2003. Large vocabulary sign language recognition based on hierarchical decision trees. In Proceedings of the 5th international conference on Multimodal interfaces. 125-131.

[7] Weiyu Guo, Chenfei Ma, Zheng Wang, Hang Zhang, Dario Farina, Ning Jiang, and Chuang Lin. 2021. Long exposure convolutional memory network for accurate estimation of finger kinematics from surface electromyographic signals. fournal of Neural Engineering 18, 2 (mar 2021), 026027. https://doi.org/10.1088/1741$2552 / \mathrm{abd} 461$

[8] T. Haifley. 2002. Linear logistic regression: an introduction. In IEEE International Integrated Reliability Workshop Final Report, 2002. 184-187. https://doi.org/10. 1109/IRWS.2002.1194264

[9] Kaiming He, Xiangyu Zhang, Shaoqing Ren, and Jian Sun. 2016. Identity Mappings in Deep Residual Networks. In Computer Vision - ECCV 2016, Bastian Leibe, Jiri Matas, Nicu Sebe, and Max Welling (Eds.). Springer International Publishing, Cham, 630-645.

[10] Yu Hu, Yongkang Wong, Wentao Wei, Yu Du, Mohan Kankanhalli, and Weidong Geng. 2018. A novel attention-based hybrid CNN-RNN architecture for sEMGbased gesture recognition. PloS one 13, 10 (2018), e0206049.

[11] Sai Nithin R. Kantareddy, Yongbin Sun, Rahul Bhattacharyya, and Sanjay E. Sarma. 2019. Learning Gestures Using A Passive Data-Glove With RFID Tags. In 2019 IEEE International Conference on RFID Technology and Applications (RFID-TA) 327-332. https://doi.org/10.1109/RFID-TA.2019.8892224

[12] Nikhil Ketkar. 2017. Introduction to PyTorch. Apress, Berkeley, CA, 195-208. https://doi.org/10.1007/978-1-4842-2766-4_12
[13] Kyung-Min Kim and Jung-In Choi. 2019. Passengers' gesture recognition model in self-driving vehicles: Gesture recognition model of the passengers' obstruction of the vision of the driver. In 2019 IEEE 4th International Conference on Computer and Communication Systems (ICCCS). IEEE, 239-242.

[14] Alex Krizhevsky, Ilya Sutskever, and Geoffrey E Hinton. 2012. Imagenet classification with deep convolutional neural networks. Advances in neural information processing systems 25 (2012), 1097-1105.

[15] Seokwon Lee, Kihong Park, Junyeop Lee, and Kibum Kim. 2017. User Study of VR Basic Controller and Data Glove as Hand Gesture Inputs in VR Games. In 2017 International Symposium on Ubiquitous Virtual Reality (ISUVR). 1-3. https: //doi.org/10.1109/ISUVR.2017.16

[16] Rung-Huei Liang and Ming Ouhyoung. 1998. A real-time continuous gesture recognition system for sign language. In Proceedings third IEEE international conference on automatic face and gesture recognition. IEEE, 558-567.

[17] Hsien-I Lin, Ming-Hsiang Hsu, and Wei-Kai Chen. 2014. Human hand gesture recognition using a convolution neural network. In 2014 IEEE International Conference on Automation Science and Engineering (CASE). IEEE, 1038-1043.

[18] Ognjan Luzanin and Miroslav Plancak. 2014. Hand gesture recognition using low-budget data glove and cluster-trained probabilistic neural network. Assembly Automation 34 (2014), 94-105. Issue 1. https://doi.org/10.1108/AA-03-2013-020

[19] Asanterabi Kighoma Malima, Erol Özgür, and Müjdat Çetin. 2006. A fast algorithm for vision-based hand gesture recognition for robot control. (2006).

[20] Meenakshi Panwar and Pawan Singh Mehra. 2011. Hand gesture recognition for human computer interaction. In 2011 International Conference on Image Information Processing. IEEE, 1-7.

[21] F. Pedregosa, G. Varoquaux, A. Gramfort, V. Michel, B. Thirion, O. Grisel, M. Blondel, P. Prettenhofer, R. Weiss, V. Dubourg, J. Vanderplas, A. Passos, D. Cournapeau, M. Brucher, M. Perrot, and E. Duchesnay. 2011. Scikit-learn: Machine Learning in Python. Fournal of Machine Learning Research 12 (2011), 2825-2830.

[22] D.L. Quam. 1990. Gesture recognition with a DataGlove. In IEEE Conference on Aerospace and Electronics. 755-760 vol.2. https://doi.org/10.1109/NAECON.1990. 112862

[23] Siddharth S Rautaray and Anupam Agrawal. 2011. Interaction with virtual game through hand gesture recognition. In 2011 International Conference on Multimedia, Signal Processing and Communication Technologies. IEEE, 244-247.

[24] K Martin Sagayam and D Jude Hemanth. 2017. Hand posture and gesture recognition techniques for virtual reality applications: a survey. Virtual Reality 21, 2 (2017), 91-107.

[25] Sungho Shin and Wonyong Sung. 2016. Dynamic hand gesture recognition for wearable devices with low complexity recurrent neural networks. In 2016 IEEE International Symposium on Circuits and Systems (ISCAS). IEEE, 2274-2277.

[26] Karen Simonyan and Andrew Zisserman. 2014. Very Deep Convolutional Networks for Large-Scale Image Recognition. (9 2014). http://arxiv.org/abs/1409.1556

[27] WJ Tan, CD Wu, SY Zhao, and Shuo CHEN. 2009. Hand gesture recognition based on fourier descriptor-BP neural network. fournal of Northeastern University (Natural Science) 130, 9 (2009), 1232-1235.

[28] James T Townsend. 1971. Theoretical analysis of an alphabetic confusion matrix. Perception \& Psychophysics 9, 1 (1971), 40-50.

[29] J. Weissmann and R. Salomon. 1999. Gesture recognition for virtual reality applications using data gloves and neural networks. In IfCNN'99. International foint Conference on Neural Networks. Proceedings (Cat. No.99CH36339), Vol. 3. 2043-2046 vol.3. https://doi.org/10.1109/IJCNN.1999.832699

[30] Liang Zhang, Guangming Zhu, Lin Mei, Peiyi Shen, Syed Afaq Ali Shah, and Mohammed Bennamoun. 2018. Attention in convolutional LSTM for gesture recognition. In Proceedings of the 32nd International Conference on Neural Information Processing Systems. 1957-1966. 